Julia A. Snyder

\title{
Language and Identity in Ancient Narratives
}

The Relationship between Speech Patterns and Social Context in the Acts of the Apostles, Acts of John, and Acts of Philip

[Sprache und Identität in Erzählungen der Antike. Die Beziehung zwischen Sprachmustern und sozialem Kontext in der Apostelgeschichte, den Johannesakten und den Philippusakten .]

Veröffentlicht auf Englisch.

Was bedeutet es, wenn ein christlicher Autor Jesus als »den Herrn« bezeichnet? Ist es in erster Linie eine Art, eine politische

JULIA A. SNYDER

Language and Identity in Ancient Narratives

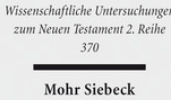

2014. XVI, 329 Seiten. WUNT II 370

ISBN 978-3-16-153330-3

DOI 10.1628/978-3-16-153330-3

eBook PDF 109,00€

ISBN 978-3-16-153264-1

fadengeheftete Broschur 109,00€ oder theologische Aussage zu treffen oder haben soziale Erwägungen mehr Einfluss auf die Wortwahl des Verfassers gehabt? Untersuchungen des frühen Christentums beruhen meistens auf einem sehr nuancierten Verständnis der lexikalischen Bedeutung, aber die neuere Forschung zieht oft soziale Aspekte der »Bedeutung der Worte« nicht in Betracht. Julia A. Snyder vertritt die Auffassung, dass methodologische Verbesserungen für die Ermittlung der lexikalischen Bedeutung in altgriechischen Texten nötig sind, die auf einer Analyse der Beziehung zwischen Sprachmustern und der Identität des Adressaten in der Apostelgeschichte, den Johannesakten und den Philippusakten gründen. Sie zeigt auch, wie soziolinguistische Variationen zur Charakterisierung und christlichen Identitätskonstruktion in den Erzählungen beitragen, wie sie die Neufassung der antiken Texte beleuchten und wie sie die Frage beeinflussen, ob die apostolischen Erzählungen für evangelistische Zwecke geschaffen wurden.

Julia A. Snyder Born 1980; 2002 BA in Classics and Mathematics; 2005 Master of Divinity; 2013 PhD in New Testament and Early Christianity at the University of Edinburgh.

Jetzt bestellen:

https://mohrsiebeck.com/buch/language-and-identity-in-ancient-narratives-9783161533303?no_cache=1

order@mohrsiebeck.com

Telefon: +49 (0)7071-923-17

Telefax: $+49(0) 7071-51104$ 